\title{
Dysregulated mitophagy and mitochondrial organization in optic atrophy due to OPA1 mutations \\ OPEN
}

Chunyan Liao, $\mathrm{PhD}$

Neil Ashley, PhD

Alan Diot, PhD

Karl Morten, PhD

Kanchan Phadwal, PhD

Andrew Williams, MD

Ian Fearnley, MD

Lyndon Rosser, BSc

Jo Lowndes, MSc, BSc

Carl Fratter, BA

David J.P. Ferguson,

$\mathrm{PhD}, \mathrm{DSc}$

Laura Vay, PhD

Gerardine Quaghebeur, MD

Isabella Moroni, MD

Stefania Bianchi, MD

Costanza Lamperti, MD, $\mathrm{PhD}$

Susan M. Downes, MD

Kamil S. Sitarz, PhD

Padraig J. Flannery, BA, MSc

Janet Carver, MSc

Eszter Dombi, BSc

Daniel East, $\mathrm{PhD}$

Matilde Laura, PhD

Mary M. Reilly, MD

Heather Mortiboys, PhD

Remko Prevo, PhD

Michelangelo

Campanella, PhD

Matthew J. Daniels, PhD, MRCP

Massimo Zeviani, MD

Patrick Yu-Wai-Man,

PhD, FRCOphth

Anna Katharina Simon,

$\mathrm{PhD}$

Marcela Votruba, PhD,

FRCOphth

Joanna Poulton, DM

\section{ABSTRACT}

Objective: To investigate mitophagy in 5 patients with severe dominantly inherited optic atrophy (DOA), caused by depletion of OPA1 (a protein that is essential for mitochondrial fusion), compared with healthy controls.

Methods: Patients with severe DOA (DOA plus) had peripheral neuropathy, cognitive regression, and epilepsy in addition to loss of vision. We quantified mitophagy in dermal fibroblasts, using 2 high throughput imaging systems, by visualizing colocalization of mitochondrial fragments with engulfing autophagosomes.

Results: Fibroblasts from 3 biallelic OPA1(-/-) patients with severe DOA had increased mitochondrial fragmentation and mitochondrial DNA (mtDNA)-depleted cells due to decreased levels of OPA1 protein. Similarly, in siRNA-treated control fibroblasts, profound OPA1 knockdown caused mitochondrial fragmentation, loss of mtDNA, impaired mitochondrial function, and mitochondrial mislocalization. Compared to controls, basal mitophagy (abundance of autophagosomes colocalizing with mitochondria) was increased in (1) biallelic patients, (2) monoallelic patients with DOA plus, and (3) OPA1 siRNA-treated control cultures. Mitophagic flux was also increased. Genetic knockdown of the mitophagy protein ATG7 confirmed this by eliminating differences between patient and control fibroblasts.

Conclusions: We demonstrated increased mitophagy and excessive mitochondrial fragmentation in primary human cultures associated with DOA plus due to biallelic OPA1 mutations. We previously found that increased mitophagy (mitochondrial recycling) was associated with visual loss in another mitochondrial optic neuropathy, Leber hereditary optic neuropathy (LHON). Combined with our LHON findings, this implicates excessive mitochondrial fragmentation, dysregulated mitophagy, and impaired response to energetic stress in the pathogenesis of mitochondrial optic neuropathies, potentially linked with mitochondrial mislocalization and mtDNA depletion. Neurology ${ }^{\circledR}$ 2017;88:131-142

\section{GLOSSARY}

DOA = dominantly inherited optic atrophy; IMM = inner mitochondrial membrane; LC3 = light chain 3; LHON = Leber hereditary optic neuropathy; MMP = mitochondrial membrane potential; $\mathbf{m t D N A}=$ mitochondrial DNA; $\mathbf{M F N 2}=$ mitofusin 2; MTOC = microtubule-organizing center; PINK1 = PTEN-induced putative kinase $1 ;$ TMRM = tetramethyl rhodamine methyl ester.

Autosomal dominant optic atrophy (DOA) is the commonest autosomal form of mitochondrial optic neuropathy, with most patients harboring pathogenic mutations in the optic atrophy 1 (OPA1) gene. OPA1 mutations cause dominantly inherited progressive visual failure in the first 2 decades, secondary to optic nerve neurodegeneration. Strikingly, a subgroup of patients develops a multisystemic neurologic phenotype, known as DOA plus. Other obligate OPA1 mutation carriers are visually asymptomatic. The mode of inheritance is autosomal dominant in the majority of cases, either haploinsufficiency or dominantnegative, with DOA plus patients frequently harboring missense mutations in the GTPase domain.

Author affiliations are provided at the end of the article.

Go to Neurology.org for full disclosures. Funding information and disclosures deemed relevant by the authors, if any, are provided at the end of the article. The Article Processing Charge was paid by the Wellcome Trust and Charity Open Access Fund.

This is an open access article distributed under the terms of the Creative Commons Attribution Licence 4.0 (CC BY), which permits unrestricted use, distribution, and reproduction in any medium, provided the original work is properly cited. 
Correspondence to Dr. Poulton:

Joanna.Poulton@obs-gyn.ox.ac.uk

Supplemental data at Neurology.org
OPA1 appears to regulate mitochondrial quality control mediated through mitophagy, ${ }^{1}$ a specialized type of autophagy. ${ }^{2}$ Mitophagy is one among several types of mitochondrial quality control, ${ }^{3}$ and the only pathway known to turn over whole mitochondrial genomes. It is crucial for normal development ${ }^{4}$ and allows dysfunctional mitochondrial DNA (mtDNA) to be recycled instead of triggering cell death. ${ }^{5}$

We previously demonstrated increased mitophagy in fibroblasts from patients with Leber hereditary optic neuropathy (LHON). ${ }^{6}$ This was attenuated by idebenone, which conferred symptomatic improvement. ${ }^{6}$ To clarify whether increased mitophagy is an important feature of mitochondrial optic neuropathies, we investigated the role of OPA1 in mitophagy in primary OPA1 mutant fibroblasts from 5 patients in 3 families with severe DOA plus phenotypes. We also studied the effects of siRNA-mediated knockdown of OPA1 in primary human control fibroblasts. Because OPA1 deficiency is widely expressed, fibroblasts have been extensively used to model the cellular mechanisms occurring in retinal ganglion and muscle cells in this multisystem disease. $^{7,8}$

METHODS Mitophagy is a sequence of events in which a structure known as the autophagosome ${ }^{9}$ forms and engulfs spent mitochondria in a process facilitated by microtubule motors. The autophagosome is then transported towards the cellular microtubule-organizing center ${ }^{10}$ (MTOC) and fuses with lysosomes, ultimately resulting in the degradation of its enclosed cargo. We therefore quantified mitophagy by counting autophagosomes, that is, characteristic puncta positive for microtubule-associated protein 1 light chain 3 (LC3), and colocalizing with mitochondrial markers. ${ }^{2}$

Standard protocol approvals, registrations, and patient consents. Ethics: Patient and control fibroblast lines. Patient and control samples were obtained with informed consent with the approval of the UK National Research Ethics Service (South Central-Berkshire and Newcastle and North Tyneside), or of the Ethical Committee of the Foundation Carlo Besta Institute of Neurology, according to the Declaration of Helsinki. Donors included 5 patients with DOA plus phenotypes, 5 other family members sharing mutant $O P A 1$ alleles, and 20 normal controls.

Pedigrees of 3 biallelic patients harboring compound heterozygous OPA1 mutations (strictly described as semi-dominant ${ }^{11-13}$ ) are presented in figure 1A. A summary of the clinical presentations and genotypes of all patients (illustrated in figure 1B) are presented in the table. This includes chronic progressive external ophthalmoplegia with an apparent defect in mtDNA maintenance $^{14,15}$ that remains unexplained (DOA plus OPA1 $[+/-] 1$ and 2, table). Further details of the clinical presentation, a cranial MRI scan of the biallelic patients, and the likely effects on protein are presented in appendix e-1 and figure e-1, A and B, at
Neurology.org. Following the convention of previous authors, ${ }^{13}$ we designated the 3 biallelic patients DOA plus because each had clinical and electrophysiologic evidence of both peripheral and optic neuropathy.

Immunofluorescence and live cell imaging. Cells were processed for histochemistry, immunofluorescence, or live staining with PicoGreen and tetramethyl rhodamine methyl ester (TMRM) as previously described (appendix e-2). We used 2 high-throughput imaging systems for detecting mitophagy: the established IN Cell $1000^{16}$ and ImageStream, which we validated (figure e-2).

Statistical analysis. Statistical analysis is detailed in appendix e-2.

RESULTS Biallelic OPA1 mutant patients and families. We studied primary fibroblasts, carrying biallelic OPA1 mutations, from patients and transmitting relatives belonging to 2 families (see table for an explanation of nomenclature, figure $1 \mathrm{~A}$ for pedigree, and appendix e-1 for additional clinical details). The proband of family 1 , DOA plus $O P A 1(-/-) 1$, is a 17 year-old boy presenting with a severe OPA1 phenotype (figure 1A). DOA plus OPA1(-/-)1 carries a c.2708_2711delTTAG p.V903Gfs*3 mutation, found in the paternal grandfather, in trans with a maternal c.661G $>$ A p.E221K change $(O P A 1[+/-] 1$ and $\mathrm{N} 1$, respectively, in figure $1 \mathrm{~A})$. In family 2, biallelic patients DOA plus OPA1 $(-/-)$ 2 and 3 both had a paternal c.2353delC p. Q785Sfs*15 and a maternal c.2869C $>$ T, p.H957Y mutation (figure $1, \mathrm{~A}$ and $\mathrm{B}$; see figure $\mathrm{e}-1 \mathrm{~B}$ for PolyPhen analysis). No other relatives were affected. The frameshift mutation in family 1 is a wellestablished pathogenic mutation. ${ }^{17}$ None of these mutations involves the GTPase domain of OPA1, classically implicated in syndromic DOA, ${ }^{13}$ examples of which were identified in monoallelic DOA plus families 3 and 4 (table).

Fibroblasts from DOA plus patients have a fragmented mitochondrial network with occasional mtDNA-depleted cells. We investigated the cellular phenotype of probands, transmitting relatives, and controls. We visualized both mtDNA and mitochondria by using the DNA-specific dye PicoGreen and the mitochondrial membrane potential (MMP)sensitive dye TMRM. ${ }^{18}$ The mitochondrial network had a fragmented morphology in a small minority of cells from patients DOA plus OPA1(-/-)1-3, but it was normal in other cells (figure $1 \mathrm{C}$ ). Using highthroughput imaging (figure 1D), we showed that mitochondria in fibroblasts from biallelic and monoallelic DOA plus patients (DOA plus OPA1 $[-/-] 1-3$ and DOA plus OPA1 $[+/-] 1-2)$ were significantly more fragmented than mitochondria from 6 controls $(p=0.005$ and 0.01 , respectively, figure e-3A). Using PicoGreen to visualize mtDNA, ${ }^{19}$ we found a significant increase in cells that were 


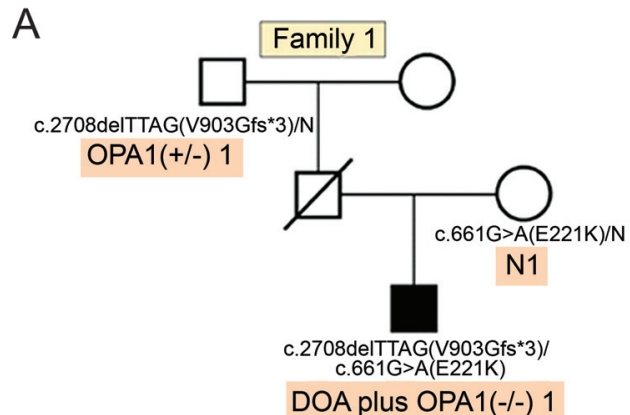

DOA plus OPA1(-/-) 1

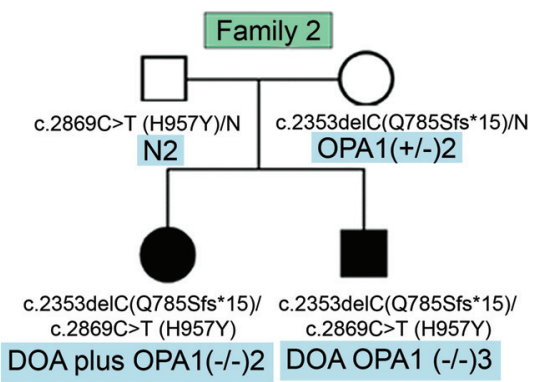

DOA plus OPA1(-/-)2 DOA OPA1 (-/-)3

B

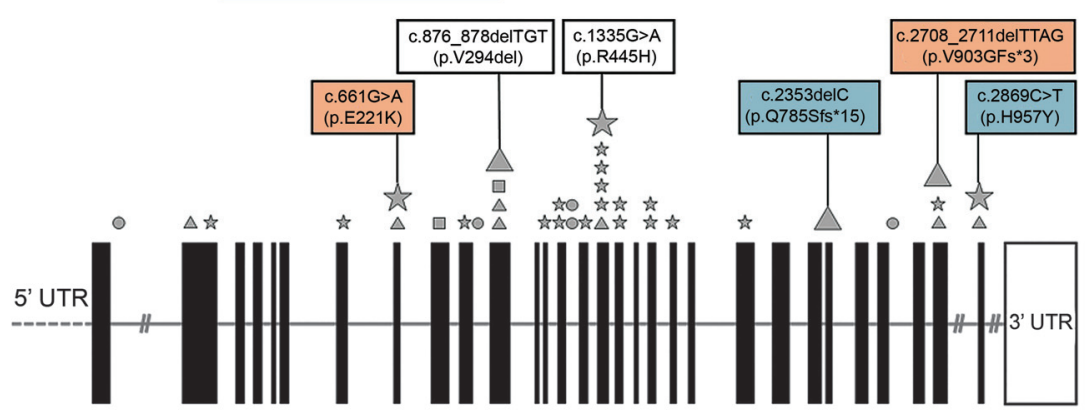

$$
\text { Exons }\left\{\begin{array}{lllll}
1 & 2 & 3 & 4 b^{5}
\end{array}\right.
$$

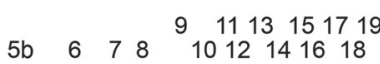

$202121^{23} 24^{25} 26^{27} 28$

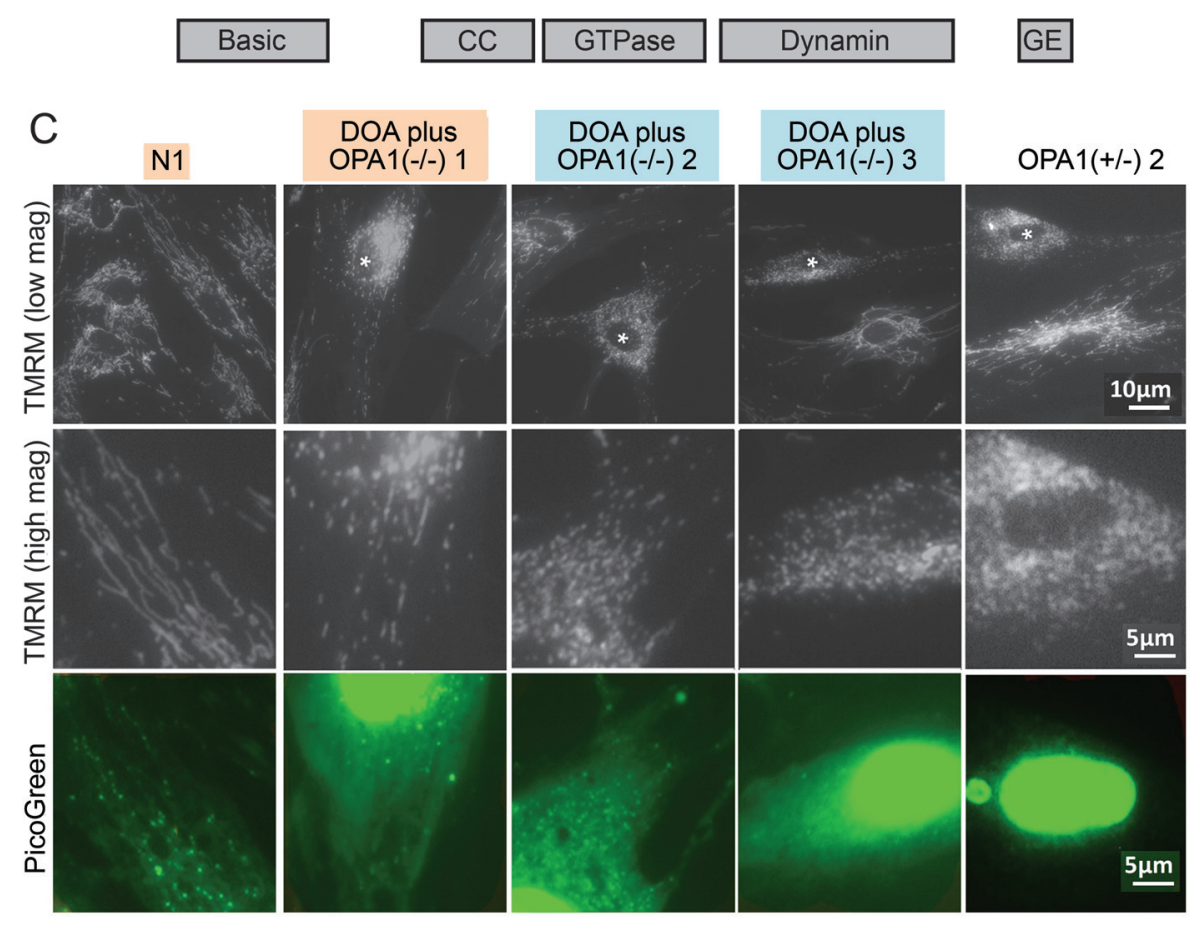

E.a. Cells depleted

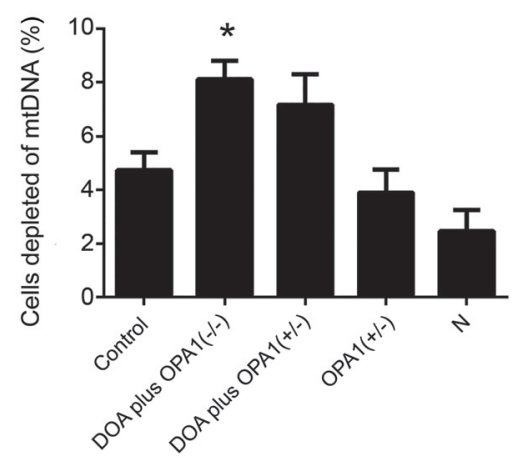

E.b. TMRM staining

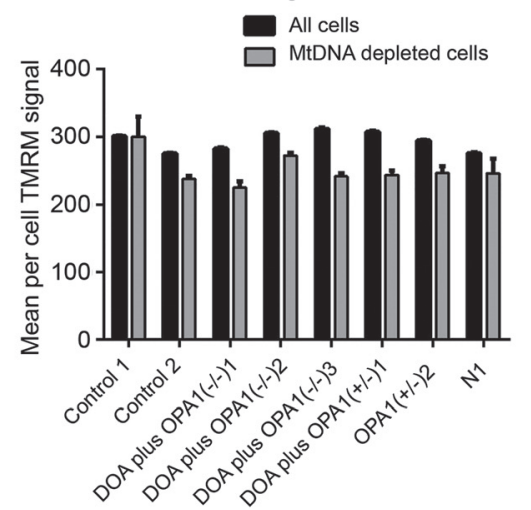

(A) Pedigrees of families 1 and 2. (B) OPA1 gene structure. Diagrammatic representation of the OPA1 gene. The diagram indicates the location both of mutations resulting in DOA plus syndromes as described ${ }^{8}$ (small symbols) and of the mutations reported in this study (large symbols; highlighting corresponds to pedigree). Mutation type: stars (missense); squares (nonsense); circles (splice site); triangles (deletion). CC = coiled-coil domain; GE = GTPase effector domain; UTR = untranslated region. (C) PicoGreen/tetramethyl rhodamine methyl ester (TMRM) costaining of live fibroblasts from biallelic DOA plus OPA1(-/-)1-3 patients, and their symptom-free mothers (N1 and OPA1[+/-]2; see A). PicoGreen stains DNA and TMRM is sensitive to mitochondrial 
depleted of mtDNA in biallelic patients compared to controls with IN Cell $1000(p<0.001$, figure 1E). In all cultures, these mtDNA-depleted cells had fragmented mitochondria with a lower membrane potential (figure 1E.a and 1E.b) than control cells. Intermediate mitochondrial fragmentation and mtDNA depletion were present in fibroblast cultures from DOA OPAI $(+/-)$ but not from non-syndromic DOA (figure e-3A) or the asymptomatic, obligate carrier relatives of the biallelic patients.

OPA1 knockdown causes mtDNA depletion and alters the distribution of mitochondria in control cells. To determine whether mitochondrial DNA depletion is a consistent effect of OPA1 knockdown ${ }^{20}$ and whether it would be sufficient to affect mitochondrial function, we then knocked down OPA1 in control fibroblasts using a pan-OPA1-specific siRNA, ${ }^{21}$ thus modeling the reduction in full-length OPA1 protein in patient cells. Compared to the reduced OPA1 protein levels seen in the patient fibroblasts, the siRNA achieved a more profound reduction (figure $2 \mathrm{~A}$ ), and knockdown cells underwent fragmentation and perinuclear clustering of the mitochondrial network (figure 2B).

Next, we visualized both mtDNA and mitochondria in the OPA1 siRNA-treated cells, ${ }^{18}$ and found a marked loss of mtDNA (figure 2C). In these cells, mitochondria clustered in the perinuclear region (figure 2, B-D), and often displayed high TMRM fluorescence, suggesting increased MMP or increased organelle density. We confirmed these findings using anti-DNA immunoglobulin M/MitoTracker colabeling of mtDNA (figure 2C) and real-time PCR (figure 2E). Despite the considerable mtDNA depletion, COX activity was largely preserved at 5 days, but reduced by 14 days (figure 2D).

By using an antibody against pericentrin, we showed that the perinuclear mitochondrial clusters consistently colocalized with the MTOC (figure 2F.a). As well as being crucial for neuronal survival and function, microtubule-dependent transport mediates efficient encounters of autophagosomes with lysosomes, ${ }^{22}$ which cluster near the nucleus under conditions such as nutrient deprivation. ${ }^{15,23} \mathrm{~A}$ similar clustering of mitochondria occurs by overexpressing tau, ${ }^{24}$ because tau inhibits microtubule-dependent plus-end-directed transport of mitochondria. Thus, we hypothesized that clustering of mitochondria at the MTOC in knockdown cells may be due to either decreased plus-end or increased minus-end transport caused by excessive fragmentation and mitophagy. To test this idea, we exposed cells to microtubuledisrupting drugs. Nocodazole, which disassembles microtubules, rescued the perinuclear clustering so that the distribution of mitochondria resembled that in control cells (figure 2F.b). Exposure to taxotere (disrupts MTOC) and cytochalasin D (depolymerizes actin) disrupted perinuclear mitochondrial clustering, supporting our assertion that it depends on microtubules and MTOC. For a more detailed explanation, see figure e-3B. Together, these results demonstrate that OPA1 knockdown in primary human fibroblasts causes disruption of the mitochondrial network, partial mtDNA depletion, and microtubule-dependent rearrangement of the mitochondrial distribution.

High-throughput imaging shows that patient fibroblasts harbor increased autophagosomes colocalizing with mitochondria compared to controls. We reasoned that the depletion of mtDNA associated with OPA1 knockdown could be due either to slowed mtDNA synthesis or to increased mtDNA turnover and therefore investigated whether OPA1 insufficiency/ dysfunction had affected mitophagy. We measured total mitochondrial autophagy irrespective of Parkin and PINK1 using 2 high-throughput imaging systems, ImageStream and IN Cell 1000, ${ }^{16}$ which are established methods for quantifying autophagy and mitophagy. In each of these, antibodies to LC3 and Tom20 are used to immunolabel autophagosomes and mitochondria, respectively. In figure e-2D, we show that ImageStream and IN Cell 1000 techniques are comparable.

Fibroblasts from DOA plus OPA1(-/-)2 and 3 (figure 3A.a) and DOA plus OPA1(-/-)1 (figure 3A.b and 3A.c) patients all harbored significantly more LC3-positive puncta colocalizing with mitochondrial fragments, and hence more mitophagy than those from the control using ImageStream. Colocalization of the lysosomal marker, LyosID, with LC3 puncta is used to demonstrate autolysosomes, a later stage of mitophagy than autophagosomes (figure 3A.a

Figure 1 legend, continued:

membrane potential. Nuclei of cells exhibiting mitochondrial fragmentation are marked with an asterisk. PicoGreen panel shows the same field as the highmagnification TMRM panel. TMRM staining of cells from biallelic DOA plus patients with abnormal mitochondrial fragmentation were often also depleted of mtDNA (E), but this was more marked in the siRNA-treated cell cultures in figure 2. (D) We used IN Cell 1000 to measure the mean mitochondrial length in fibroblast cultures, stained either with TMRM and PicoGreen ${ }^{25,26}$ or with antibody to mitochondrial protein Tom20. Cultures were grown for 3 days in 96 -well plates in triplicate. To quantify the degree of mitochondrial fragmentation, we measured the average mitochondrial length in each cell and plotted a frequency distribution. This shows that while the modal length was similar in both groups, the per cell average mitochondrial length was shorter in biallelic patients (D.a) than controls (D.b) (see also figure e-3). (E) Cells depleted of mtDNA are increased (E.a) and have a lower membrane potential by TMRM staining (E.b). Error bars are 1 standard error. Asterisks indicate $p<0.001$ compared to controls (2-tailed $t$ test). Each bar represents between 400 and 1,500 cells. mtDNA = mitochondrial DNA. 


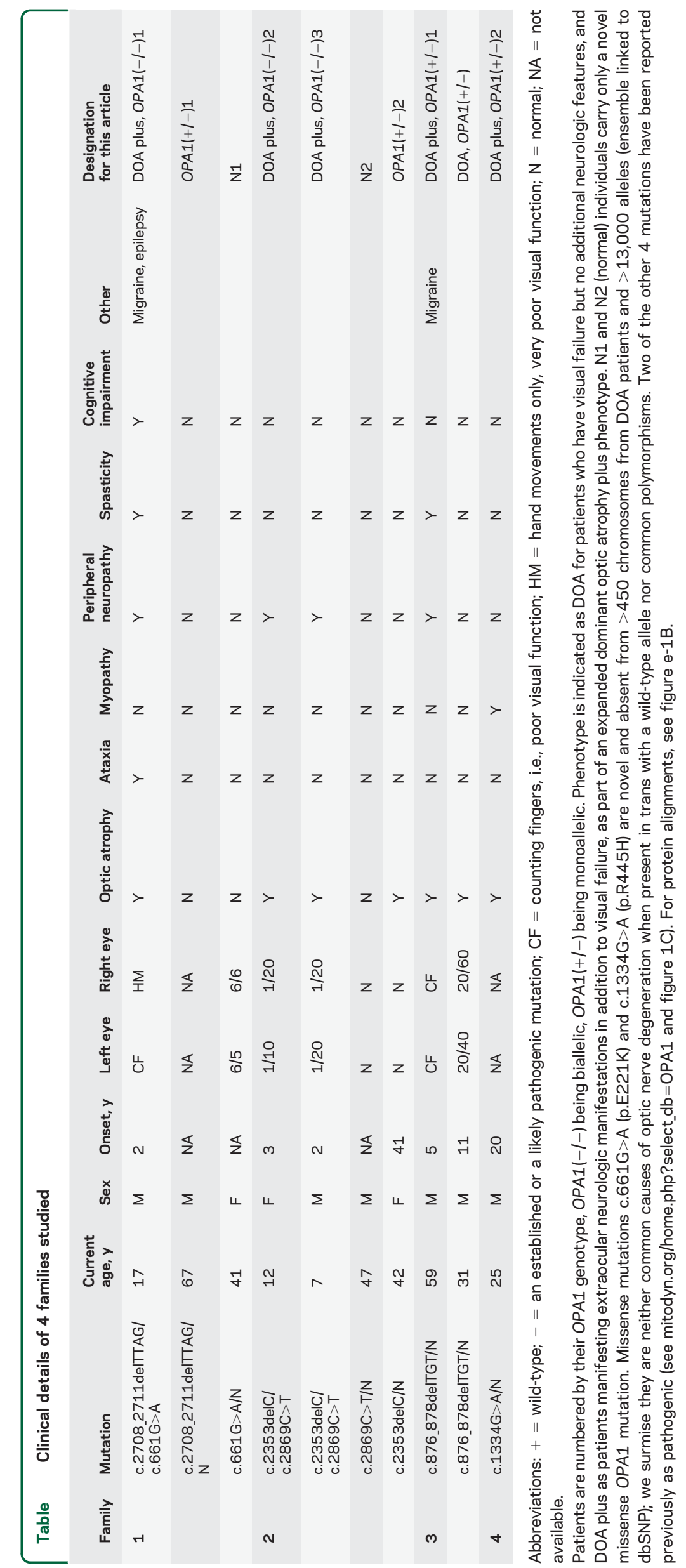

and 3A.b, respectively). Increased colocalization of mitochondria with LC3/LyosID-positive autolysosomes supported an increase in mitophagy in these biallelic patients (figure 3A.c). Figure 3B shows that the increase in mean level of mitophagy in the group of all DOA plus patients (combining biallelic and monoallelic) compared to controls over 4 independent experiments was significantly increased ( $p=$ 0.035 ). It was not increased in nonsyndromic monoallelic relatives. Analysis of control fibroblasts treated with OPA1 siRNA also suggested that mitophagy was increased compared with scramble siRNA (figure 3C.a). This is consistent with the increase in LC3-II abundance on Western blot analysis (figure 3C.b).

Similarly, quantitative fluorescence microscopy using IN Cell $1000^{16}$ confirmed that LC3 puncta colocalizing with mitochondria were increased in cells from biallelic patients at baseline, compared to controls (figure 3D). Similar increases in basal mitophagy were seen in fibroblasts from 2 monoallelic DOA plus OPA1(+/-) patients who had GTPase domain mutations (DOA plus OPA1 $[+/-] 1$ and DOA plus OPA $[+/-] 2)$, but were comparable to control levels in cells from 5 individuals who had monoallelic OPA1 mutations (N1, $\mathrm{N} 2, \quad O P A 1[+/-] 1, \quad O P A 1[+/-] 2$, and DOA $O P A 1[+/-])$.

Mitophagic flux is increased in fibroblasts from biallelic DOA plus patients. An increase in autophagosomes could reflect either increased autophagic activity or a reduced turnover; we therefore measured mitophagic flux. This is defined as the ratio of the magnitude of the increase in counts of puncta colocalizing with mitochondria over basal levels, relative to basal mitophagy, ${ }^{2}$ in a range of culture conditions and in the presence of lysosomal inhibitors. Growing fibroblasts on starvation (culture in minimal medium) or glucose-free galactosebased media (henceforth galactose medium) forces mitochondria to use oxidative phosphorylation and increases mitophagy. ${ }^{16}$ These culture conditions both generated a greater increase in colocalizing puncta in biallelic patients than in controls on both ImageStream (figure 3A) and IN Cell 1000 (figures $3 \mathrm{D}$ and $4 \mathrm{~B}$ and not shown). Lysosomal inhibitors had a similar effect (figures 3, A, B, and $\mathrm{D}$, and $\mathrm{e}-4$ ).

Such conditions may also activate autophagy, consistent with the increase in LC3-ll seen on Western analysis of cells cultured in galactose (figure e-5), but this increase is less reproducible.

Effect of OPA1 mutations on mitophagy is modulated by knocking down proteins involved in mitophagy. To confirm that the increased colocalization of LC3 puncta 


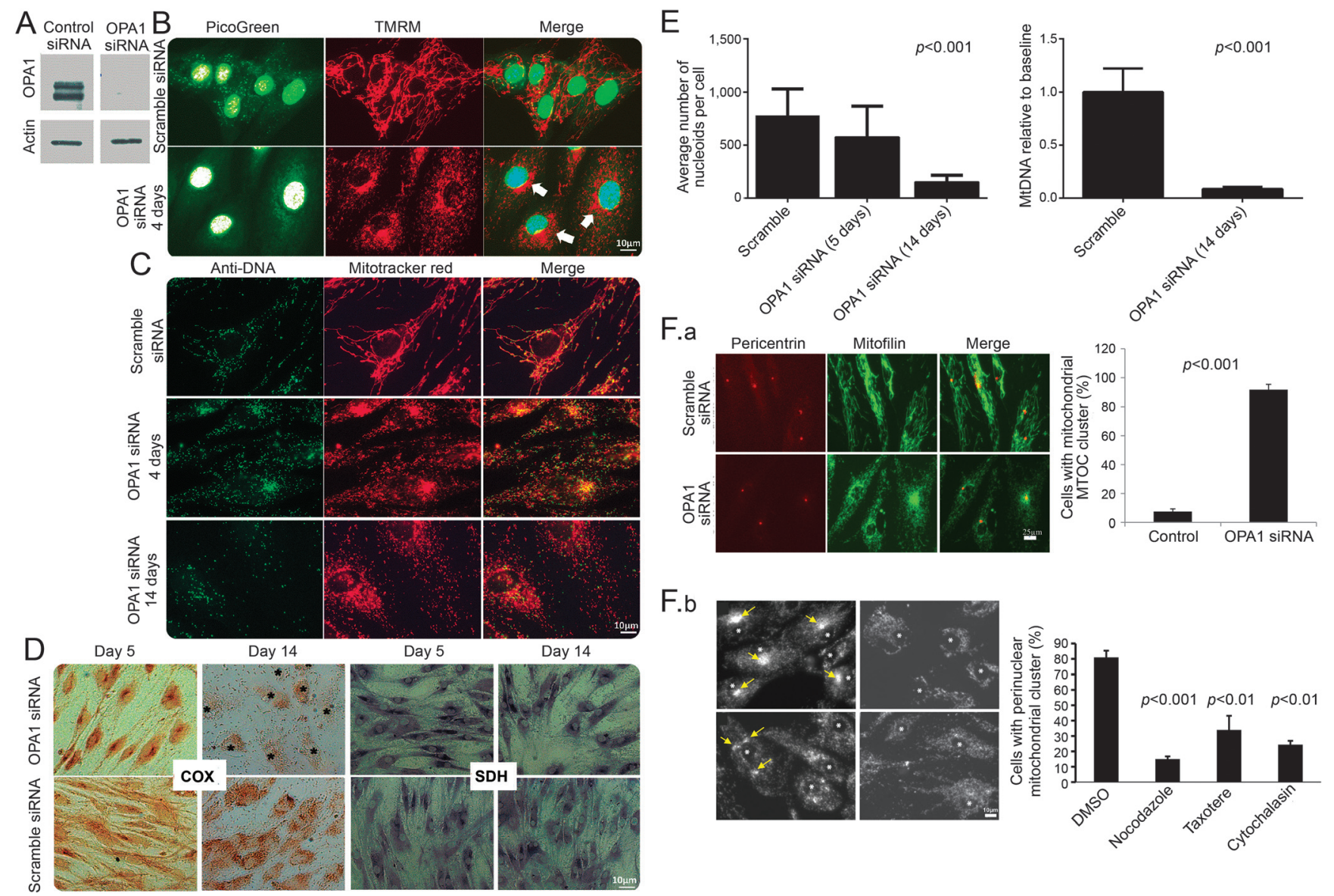

Control fibroblasts were treated with OPA1 Pan OPA1 or scramble siRNA. (A) Western blot analysis confirms OPA1 levels were efficiently reduced following siRNA treatment (blot shows 3 days after knockdown). (B) PicoGreen/tetramethyl rhodamine methyl ester (TMRM) costaining of live fibroblasts treated with OPA1 siRNA shows a marked loss of mtDNA nucleoids (visible as green puncta) compared to treatment with scramble. In all OPA1 knockdown cells with mitochondrial fragmentation, a noticeable mitochondrial perinuclear clustering was observed (arrows). (C) Anti-DNA/MitoTracker colabeling of OPA1/ scramble siRNA treated fibroblasts (note that this antibody is recognized to label mtDNA more strongly than nuclear DNA). This confirms the findings presented in B. (D) Cytochrome oxidase (COX) activity, demonstrated by routine histochemistry, is significantly reduced by day 14 . In the panel showing COX at 14 days in the knockdown, the positions of nuclei are marked with asterisks. By day 5, the distribution of both $\mathrm{COX}$ and succinate dehydrogenase (SDH) activity reflect the mitochondrial perinuclear clustering. Figure e- 6 shows that mitochondrial fragmentation occurred without loss of cytochrome $c$ or alteration of cristae at 48 hours. (E) SiRNA to OPA1 significantly reduced the number of nucleoids visualized by PicoGreen (left panel, $p<0.001,2$-tailed $t$ test) corresponding to $m t D N A$ content of $8 \% \pm 2 \%$ of the control. This was confirmed by quantitative PCR 14 days after treatment (right panel, $p<0.001$, 2-tailed $t$ test). (F) Cell biological basis for relocalization of mitochondria during OPA1 knockdown implies altered transport of fragmented mitochondria. OPA1 knockdown relocates mitochondria to the microtubule organizing center (MTOC), mediated by microtubules and actin. That this is prevented by overexpression of p50-dynamitin is shown in figure e-3B. (F.a) Anti-pericentrin (MTOC)/mitofillin (mitochondria) staining shows that siRNA to OPA1 causes relocation of mitochondria to the MTOC in over $90 \%$ of cells as opposed to the scrambled siRNA control (see chart). (F.b) The cell biological basis for relocalization of mitochondria during OPA1 knockdown implies altered transport mediated by microtubules and actin. Upper left: confirms the presence of perinuclear mitochondrial clusters following OPA1 knockdown. Upper right: Disassembly of microtubules by nocodazole treatment ( $5 \mu \mathrm{M}, 24$ hours) rescues the mitochondrial clustering ( $<0.001)$. Lower left: Taxotere $(5 \mu \mathrm{M}, 24$ hours) causes assembly and stabilization of microtubules and hence randomly distributed mitochondrial clusters ( 3 clusters in one cell each marked with an arrow, $p<0.01$ ). Lower right: Treatment with cytochalasin $\mathrm{D}(5 \mu \mathrm{M}, 24$ hours), which depolymerizes actin, also redistributes mitochondria away from the MTOC, although a weak perinuclear clustering was still present ( $p<0.01)$. Each of these experiments was carried out at least 3 times.

with mitochondria involved mitophagy, we knocked down the essential autophagy protein ATG7 ${ }^{4}$ (figure $4 \mathrm{~A})$. We therefore performed RNAi on fibroblasts from DOA plus OPA1(-/-)1-3 patients and controls, obtaining a good reduction in ATG7 protein levels (figure 4A). Both total and colocalizing LC3 puncta were reduced by $A T G 7$ knockdown in all conditions $(p<0.001$, figure $4 \mathrm{~B})$, eliminating the difference between biallelic patients and controls, both at baseline and after addition of the lysosomal inhibitors E64D and pepstatin A.

Effect of idebenone. Exposure of fibroblasts to idebenone, which modulates the increased mitophagy that we demonstrated in LHON, ${ }^{6}$ had no effect (figure e-4B).

A mitofusin 2 mutation increases mitochondrial fragmentation and mitophagy. Mitochondrial depolarization and ubiquitination are accepted triggers 
Figure 3 Analysis of primary cultures from biallelic dominantly inherited optic atrophy (DOA) plus OPA1(-I-) patients demonstrates increased mitophagy compared to an age-matched control

A.a

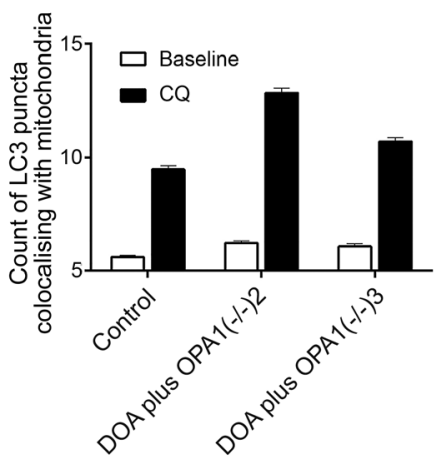

B

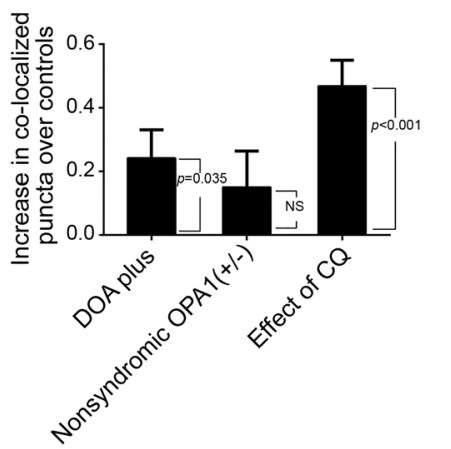

D.a

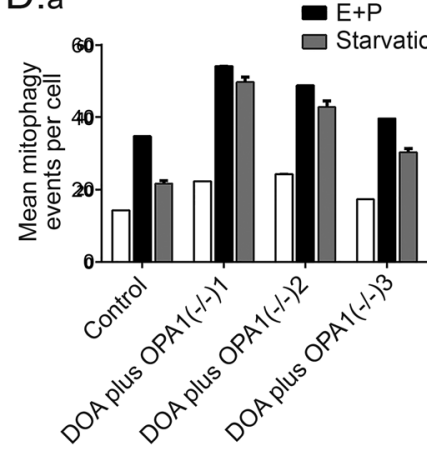

A.b

$\square$ Control

DOA plus OPA1(-/-)1

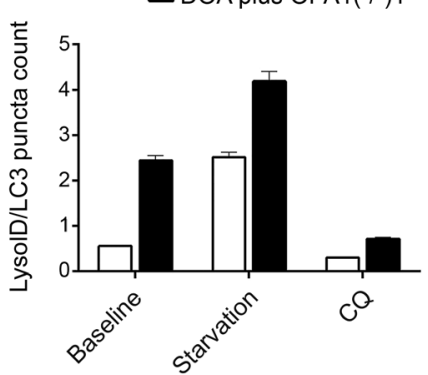

C.a

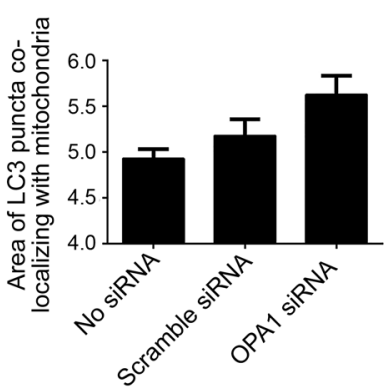

D.b

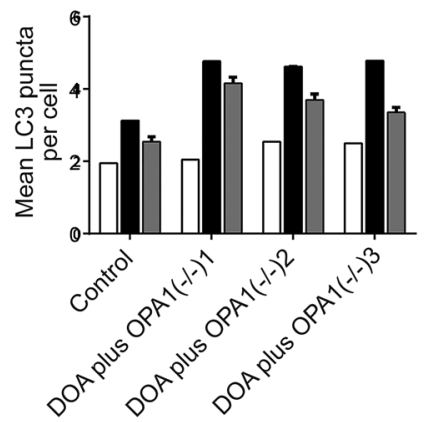

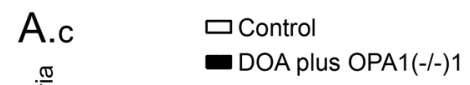

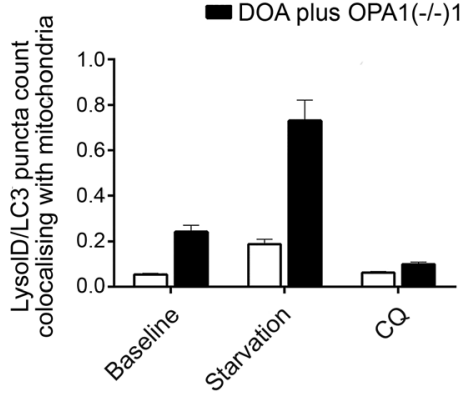

C.b No Scramble OPA1

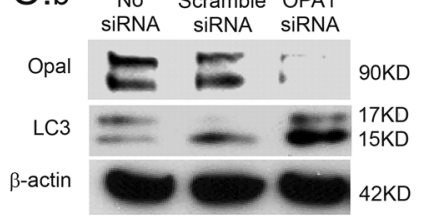

D.c

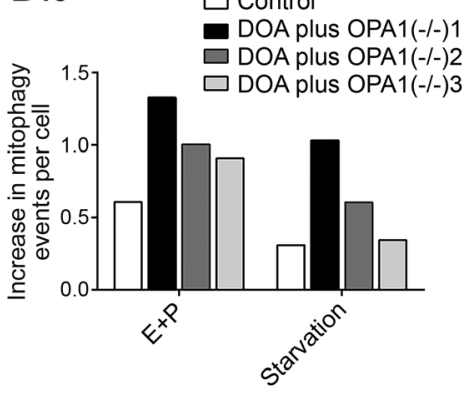

(A) ImageStream analysis of cultured fibroblasts shows that basal mitophagy is significantly increased in DOA plus OPA1 $(-I-) 2$ and DOA plus OPA1 (-I-)3 compared with control both at baseline and following treatment with CQ (all $p<0.01,2-$ tailed $t$ test). (A.a) The number of puncta per cell that were positive for light chain 3 (LC3) (representing both autophagosomes and autolysosomes) and Tom20 (representing mitochondria). These were counted at baseline in control cells, for comparison with patients DOA plus OPA1(-I-)2 and 3. Exposure to chloroquine (CQ; $25 \mu \mathrm{M}$ ) overnight blocks mitophagy at this stage by preventing lysosomal acidification increasing the signal, more so in patients than controls. (A.b, A.c) The counts of puncta that are positive for both LC3 and LysoID (representing only autolysosomes), counted in control cells, for comparison with patient DOA plus OPA1(-I-)1. (A.b) The total number of puncta per cell that were positive for the autolysosome markers. (A.c) The counts of these autolysosomes that colocalized with mitochondria (hence autolysosomes involved in mitophagy) for the same dataset. In all cases there were more counts in the patient than the control. Galactose-based starvation medium increased the number of LC3/LysolD-positive puncta above baseline. Exposure to $25 \mu \mathrm{M} C \mathrm{CQ}$ overnight did not increase the signal, because it prevents progression of autophagosomes to autolysosomes. Error bars are standard errors (SEs) (technical replicates). Patient values all significantly greater than control $p<0.01$ (2-tailed $t$ test). All are representative of 1 out of 3 independent replicates. (B) A statistical analysis of 4 consecutive ImageStream runs on all the patients listed in the table along with 6 controls. The output shows increased mitophagy in patients with severe OPA1 mutations (both biallelic and monoallelic, that is, DOA plus OPA1[-I-] and DOA plus OPA1[+l-]) compared with normal controls ( $p=0.035)$. We show one bar per patient group, with each bar's height (y axis) representing the estimated difference between a particular patient group and controls. The whiskers on a bar represent the SE of the estimated difference ( \pm 1 SE is shown); an approximate $95 \%$ confidence interval for the patient-control difference could be calculated as the bar height \pm 2 SEs. The $p$ values in the figure are from the test of the null hypothesis that there is no actual difference 
for mitophagy, in some situations mitophagy being amplified by ubiquitinylation of the outer membrane proteins, mitofusin 1 and $2,{ }^{25}$ by Parkin, a ubiquitin ligase recruited to depolarized mitochondria in connection with PTEN-induced putative kinase 1 (PINK1). ${ }^{26,27}$

Neither mitochondrial depolarization nor ubiquitination were apparent in our patient fibroblasts (figure $1 \mathrm{E}$ and not shown), so we questioned whether mitochondrial fragmentation was sufficient in itself to trigger mitophagy. We therefore studied fibroblasts from a patient with a dominant negative mutation in another mitochondrial pro-fusion gene, mitofusin 2 (MFN2). These fibroblasts showed increased fragmentation of mitochondria compared to controls $(p=0.05)$, associated with increased mitophagy, both at baseline and after treatment with the lysosomal inhibitor, chloroquine $(p<0.02$ and 0.001, respectively, figure $4 \mathrm{C}$ ).

DISCUSSION We showed that profound loss of OPA1 has several effects beyond mitochondrial fragmentation that potentially contribute to the pathogenesis of DOA and the onset of clinical disease. These include increased mitophagy, mitochondrial mislocalization, and, potentially, mitochondrial dysfunction due to mosaic mtDNA depletion.

We identified 3 patients who each carried one frameshift mutation in trans with a novel missense mutation, designated biallelic OPA1. The term Behr syndrome has been used for other biallelic OPAI families with severe phenotypes in which a missense allele, described as hypomorphic, occurs in trans with a pathogenic allele. ${ }^{28}$ Furthermore, both frameshift mutations caused nonsyndromic DOA with incomplete penetrance, yet caused DOA plus when combined with a missense mutation.
OPA1 is a transmembrane protein embedded within the inner mitochondrial membrane (IMM), involved in mitochondrial dynamics, specifically in IMM fusion ${ }^{29}$ and maintenance of cristae. It is protective against apoptosis ${ }^{30}$ and neurodegeneration. ${ }^{31}$ Mutant cells derived from patients with biallelic OPA1 mutations not only had a lower level of OPA1 protein, but there was evidence of significant mitochondrial fragmentation compared with controls (figure 1D). A small proportion of these cells with fragmented mitochondria were profoundly depleted of mtDNA (figure 1, C and E). High-throughput quantitative imaging revealed that mitochondrial fragmentation and mtDNA depletion was also increased in monoallelic DOA plus patients with dominantly inherited OPA1 mutations involving the GTPase domain. While OPA1 depletion is known to cause mtDNA depletion in neurons, ${ }^{32}$ the association in fibroblasts is novel. In line with other investigators, fragmentation and mtDNA depletion (figure 1E) were not present in fibroblast cultures from nonsyndromic DOA patients, from the asymptomatic, obligate carrier relatives of biallelic patients, or from the controls (table).

Previous investigators found that cultured cells with even severe respiratory chain defects appear to experience rather small increases in mitophagy ${ }^{33}$ and that defects in respiratory chain function, if present in OPA1 patients, ${ }^{14}$ are subtle. ${ }^{7}$ We suggest that these subtle defects may reflect the increased level of mtDNA-depleted mitochondria in cells that we documented. Two high-throughput imaging systems (ImageStream and IN Cell 1000) provide objective evidence of increased colocalization of mitochondria with autophagosomes and autolysosomes. These are more sensitive and specific for measuring mitophagy than conventional fluorescence and electron microscopy and Western blotting. Both methods showed

Figure 3 legend, continued:

between a patient group and controls. Useful intuition connecting the hypothesis test with the estimated difference is that a p value $<0.05$ corresponds to a $95 \%$ confidence interval not overlapping zero. Uncomplicated symptomatic DOA OPA1 $(+/-)$ and asymptomatic OPA1(+I-) were not different from controls ( $n=1,2$, and 6, respectively). Chloroquine $25 \mu \mathrm{M}$ overnight CQ significantly increased the number of LC3 puncta colocalizing with the mitochondrial signal in all individuals in all experiments $(p<0.001$ ). (C) OPA1 knockdown by siRNA also increases mitophagy. (C.a) Bar chart of ImageStream output shows that siRNA to OPA1 increases mitophagy. The summed area of LC3 puncta that colocalize with mitochondria (PDH signal) in fibroblasts treated with OPA1 siRNA is greater than in scramble siRNA and the untreated controls $(p=0.05$ and $p<0.01$, respectively, both 2 -tailed $t$ tests). Mitochondrial mean intensity was also reduced by $5 \%$ (not shown). Error bars are SEs (technical replicates). (C.b) In OPA1 knockdown fibroblasts (OPA1 siRNA) compared to untreated (No siRNA) and siRNA scramble (Scr siRNA) controls, OPA1 levels are reduced and LC3-II levels are substantially increased relative to actin by the Western blot analysis. (D) Validation of the increased mitophagic flux, using IN Cell 1000 quantitative fluorescence microscopy. The number of LC3-positive puncta per cell was quantitated in fibroblasts from biallelic OPA1 patients at baseline and after 2 hours in the presence of lysosomal protease inhibitors (E64D and pepstatin A, labeled E+P) or after 2 hours starvation in minimal medium compared with age-matched controls. (D.a) Per cell count of LC3-positive puncta colocalizing with mitochondria ( $p<0.0001$ and $p<0.005$ for baseline compared to lysosomal inhibitors or starvation, respectively). (D.b) Mean number of total LC3-positive puncta per cell. Each patient had significantly higher counts than control $(p<0.02)$ in all conditions, except baseline patient DOA plus OPA1 $(-\mid-) 3$ autophagy and baseline patient DOA plus OPA1(-/-)1 mitophagy. (D.c) The mitophagic flux is increased in biallelic patients relative to controls (the increase in colocalization during starvation or lysosomal inhibitors, relative to baseline). Error bars are SEs of technical replicates, all $p$ values 2 -tailed $t$ tests. For further evidence of increased mitophagic flux, see figure e-4A. 
Figure 4 Effect of OPA1 mutations on mitophagy is impaired by knockdown of ATG7 and recapitulated by a mitofusin 2 (MFN2)-dominant negative genotype

A
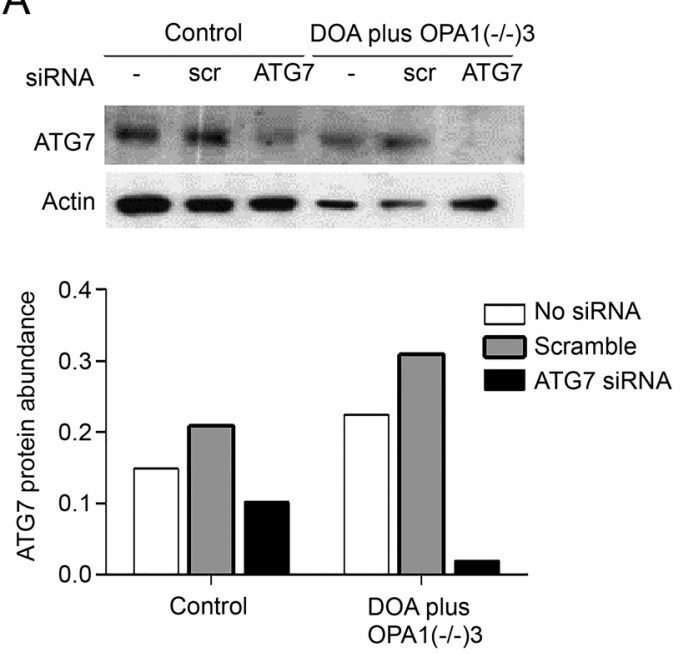

\section{C.a. Baseline}

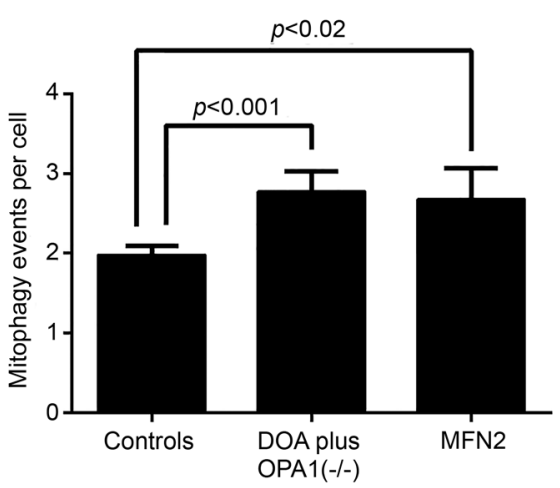

B.a. Baseline
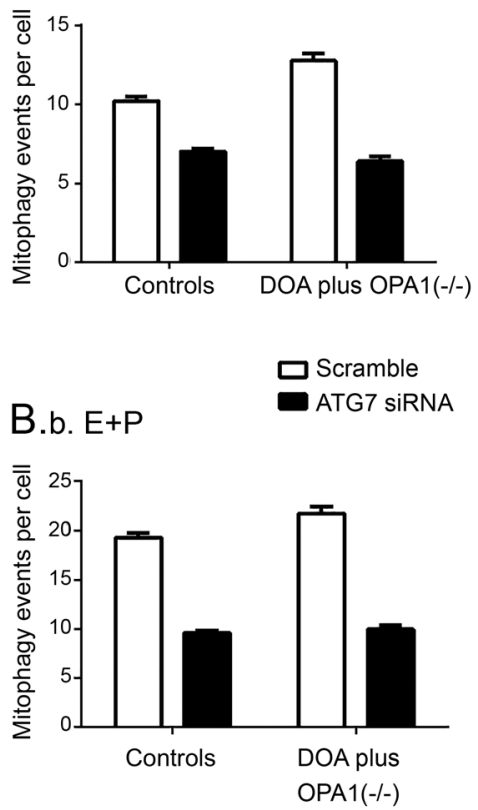

C.b. Chloroquine-treated

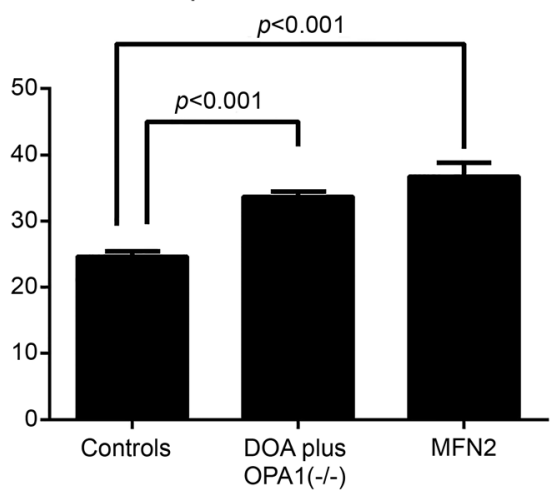

(A) Western blot analysis confirms that knockdown of ATG7 by siRNA reduced protein abundance relative to actin in both patient and control cells. ATG7 = siRNA to ATG7; DOA = dominantly inherited optic atrophy; scr = scramble; siRNA = no treatment. (B) The number of colocalizing mitochondrial puncta (mitophagy events per cell, measured by IN Cell 1000) is significantly increased in fibroblasts from biallelic patients compared to controls at baseline (B.a) $(p<0.001)$ and after blocking autophagy with lysosomal inhibitors $E 64 D$ and pepstatin $A(E+P, p=0.01)$. (B.b) Adding E64D and pepstatin $A$ significantly increased the numbers of colocalizing puncta $(p<0.001)$ compared to baseline. Knockdown of ATG7 protein by siRNA significantly reduced the colocalizing puncta $(p<0.001)$ and eliminated the increase in patients over controls. Cells were treated with siRNA against ATG7 for 48 hours, then the medium was changed and either solvent or lysosomal inhibitors, E64D, and pepstatin A were added for 3 days. Error bars are standard errors of technical replicates, all $p$ values 2-tailed $t$ tests. (C) The number of mitophagy events per cell (measured by IN Cell 1000) at baseline (C.a) is significantly increased in fibroblasts from biallelic DOA plus OPA1 $(-I-)$ patients $(\mathrm{n}=3)$ and in a patient with a dominant negative MFN2 mutation compared to control ( $p<0.001$ and $p<0.02$, respectively). After adding chloroquine (C.b), this was significantly increased in both the DOA plus OPA1 $(-\mid-)$ patients $(n=3)$ and the MFN2 fibroblasts compared to control (both $p<0.001)$. In neither case was the MFN2 significantly different from biallelic DOA plus OPA1(-l-) patients. Error bars are standard errors of technical replicates, all $p$ values 2 -tailed $t$ tests.

that mitophagy is increased at baseline and following activation of autophagy in biallelic DOA plus fibroblasts, and is reduced by knockdown of the autophagy protein ATG7 (figures 4, A and B, and e-2E). The increased colocalization of mitochondria and autophagosomes represents increased mitophagic flux (figure e-4). Mitophagy was thus clearly increased in patients with monoallelic DOA plus and in severely affected biallelic OPA1 patients, but not significantly in our monoallelic unaffected participants or in mildly affected, nonsyndromic monoallelic OPA1 patients. The abundance of OPA1 protein 
reflected these differences (figure e-5). This is supported by electron microscopic findings in 2 mouse models. ${ }^{17,34}$

Because mitophagy does not appear to increase bulk turnover of all mitochondrial components, ${ }^{35}$ its importance has been called into question. It is the only type of mitochondrial quality control known to turn over whole mitochondrial genomes. While it is not clear that OPA1 mutations directly cause $\mathrm{mtDNA}$ mutations or depletion, altering the dynamic cycle of mitochondrial fission and fusion is likely to dysregulate mitophagy and impair mitochondrial quality. ${ }^{36}$

Our data show that active mitophagy closely reflects the phenotypic severity of DOA plus due to OPA1 depletion (figures 1E, 3B, and e-5). We suggest 3 ways in which these could be linked (figure e-7).

First, the increased mitophagy may be driven by an excess of fragmented mitochondria, potentially because of a respiratory chain defect that we did not detect. This could be beneficial or neutral. This increase is consistent with type 1 mitophagy, ${ }^{37}$ a subtype that is independent of PINK1 and Parkin. ${ }^{37}$ This is because we found no evidence of increased ubiquitination (not shown) and no recruitment of the mitophagy proteins PINK1 and Parkin. It is thus plausible that increased fragmentation drives type 1 mitophagy.

Further, microtubule-dependent clustering of mitochondria, which is also apparent in MFN2 knockdown, ${ }^{38}$ may also disadvantage the cell, representing a mitophagic traffic jam. For instance, clustering of fragmented mitochondria may mechanically obstruct axonal transport of functioning mitochondria or prevent mitochondrial responses to stress (stress-induced mitochondrial hyperfusion ${ }^{39}$ ).

Third, activated mitophagy may increase turnover of mitochondria and mtDNA. We showed that profound OPA1 knockdown in control fibroblasts causes progressive loss of mtDNA and eventually mitochondrial function (figure 2E). Mitophagy may be excessive in retinal ganglion cells of OPA1 patients, perhaps increasing demand on lysosomal pathways or causing mtDNA depletion in key locations. Indeed, OPA1 depletion recapitulates the effects of the mitophagy-activating drug, phenanthroline. By disrupting OPA1 processing, this metalloprotease inhibitor activates mitophagy excessively, depleting mitochondria and mtDNA and impairing the selectivity for damaged mtDNA. ${ }^{16}$

The interplay between these mechanisms remains to be determined (figure e-7). We showed evidence that OPA1 depletion affects mitochondrial fragmentation, quality control, and likely microtubular transport, all important determinants of mitochondrial mass, ${ }^{40}$ neuronal maturation, ${ }^{32}$ and health. ${ }^{3}$ These could underline the known effects of $O P A 1$ depletion on neural maturation, ${ }^{32}$ leading to retinal ganglion cell loss, optic nerve degeneration, and hence visual failure. In particular, increased mitophagy is implicated in both LHON and syndromic parkinsonism caused by OPA mutations. ${ }^{8}$ These add biological credibility to our suggestion that dysregulated mitophagy is important in the pathogenesis of mitochondrial optic neuropathies. ${ }^{6}$ If so, drug modulators of mitophagy may be useful therapies for this group of disorders.

\section{AUTHOR AFFILIATIONS}

From the Nuffield Department of Obstetrics and Gynaecology (C. Liao, N.A., A.D., K.M., L.V., J.C., E.D., J.P.), The Women's Centre; Nuffield Department of Medicine (K.P., A.K.S.), NIHR Translational Immunology Laboratory Biomedical Research Centre, Oxford; Departments of Paediatrics and Ophthalmology and Virtual Academic Unit (A.W., I.F.), Northampton General Hospital; Institute of Medical Genetics (L.R.) and Cardiff Eye Unit (M.V.), University Hospital of Wales, Cardiff; Department of Clinical Genetics (J.L.) and Oxford Medical Genetics Laboratories (C.F.), Churchill Hospital; Nuffield Division of Clinical Laboratory Sciences (D.J.P.F.); Department of Neuroradiology (G.Q.), Oxford University Hospitals NHS Trust, UK; Department of Child Neurology (I.M.) and Division of Molecular Neurogenetics (C. Lamperti), Carlo Besta Neurological Institute IRCCS; Service of Neuro-ophthalmology and Eye Electrophysiology (S.B.), The Auxological Institute IRCCS, Milan, Italy; Department of Ophthalmology (S.M.D.), John Radcliffe Hospital, Oxford; Wellcome Trust Centre for Mitochondrial Research (K.S.S., P.J.F., P.Y.-W.-M.), Institute of Genetic Medicine, Newcastle University, Newcastle upon Tyne; Department of Comparative Biomedical Sciences (D.E., M.C.), The Royal Veterinary College, University of London; MRC Centre for Neuromuscular Disorders (M.L., M.M.R.), Department of Molecular Neurosciences, UCL Institute of Neurology, Queen Square, London; Sheffield Institute for Translational Neuroscience (H.M.), University of Sheffield; CRUK/ MRC Oxford Institute for Radiation Oncology (R.P.) and Division of Cardiovascular Medicine, Radcliffe Department of Medicine (M.J.D.), University of Oxford; UCL Consortium for Mitochondrial Research (M.C.), London; MRC Mitochondrial Biology Unit (M.Z.), Cambridge; Newcastle Eye Centre (P.Y.-W.-M.), Royal Victoria Infirmary, Newcastle upon Tyne, and NIHR Biomedical Research Centre at Moorfields Eye Hospital and UCL Institute of Ophthalmology, London; and School of Optometry and Vision Sciences (M.V.), Cardiff University, UK. L.V. is currently affiliated with the Molecular, Cellular and Developmental Neurobiology Department, Cajal Institute-CSIC, Madrid, Spain.

\section{AUTHOR CONTRIBUTIONS}

Chunyan Liao: acquisition and analysis of data. Neil Ashley: acquisition and analysis of data. Alan Diot: acquisition and analysis of data. Karl Morten: acquisition and analysis of data. Kanchan Phadwal: acquisition and analysis of data. Andrew Williams: clinical description of patient. Ian Fearnley: clinical description of patient. Lyndon Rosser: acquisition and analysis of data. Jo Lowndes: clinical description of patient. Carl Fratter: acquisition and analysis of data. David Ferguson: acquisition and analysis of data. Laura Vay: acquisition and analysis of data. Gerardine Quaghebeur: clinical description of patient. Isabella Moroni: clinical description of patient. Stefania Bianchi: clinical description of patient. Costanza Lamperti: clinical description of patient. Susan Downes: clinical description of patient. Kamil S. Sitarz: acquisition and analysis of data. Padraig J. Flannery: acquisition and analysis of data. Janet Carver: acquisition and analysis of data. Eszter Dombi: acquisition and analysis of data. Daniel East: acquisition and analysis of data. Matilde Laura: characterization of patient cells. Mary M. Reilly: characterization of patient cells. Heather Mortiboys: acquisition and analysis of data. Remko Prevo: acquisition and analysis of 
data. Michelangelo Campanella: acquisition and analysis of data Matthew Daniels: acquisition and analysis of data. Massimo Zeviani: critical revision of the manuscript. Patrick Yu-Wai-Man: clinical description of patient and critical revision of the manuscript. Anna Katharina Simon: critical revision of the manuscript. Marcela Votruba: critical revision of the manuscript. Joanna Poulton: supervisor role, critical revision of the manuscript, design and conceptualization of the study.

\section{ACKNOWLEDGMENT}

The authors thank the patients and their families for participating, Daniele Ghezzi for help with bioinformatics, Aviva Tolkovsky for expert advice, Rebecca Muir and Pippa Oakeshott for proofing, and Stephen Kennedy for support.

\section{STUDY FUNDING}

This work was supported by the Oxford Partnership Comprehensive Biomedical Research Centre with funding from the Department of Health's NIHR Biomedical Research Centres funding scheme, NewLife, the MRC (MR/J010448/1), the Wellcome Trust (0948685/Z/10/Z) and the Angus Memorial Mitochondrial Fund. J.P., J.L., J.C., and C.F. have salary support from the NHS Specialized Services Rare Mitochondrial Disorders Service. K.P. and A.K.S. were funded by the NIHR Biomedical Research Center Oxford. P.Y.-W.-M. is supported by a Clinician Scientist Fellowship Award (G1002570) from the Medical Research Council (MRC, UK) and receives funding from Fight for Sight (UK), the UK National Institute of Health Research (NIHR) as part of the Rare Diseases Translational Research Collaboration, and the NIHR Biomedical Research Centre based at Moorfields Eye Hospital NHS Foundation Trust and UCL Institute of Ophthalmology. The views expressed are those of the authors and not necessarily those of the NHS, the NIHR, or the Department of Health.

\section{DISCLOSURE}

C. Liao was funded by the Wellcome Trust $(0948685 / \mathrm{Z} / 10 / \mathrm{Z})$ N. Ashley reports no disclosures relevant to the manuscript. A. Diot was funded by the MRC (MR/J010448/1) and the NewLife Foundation. K. Morten was funded by the Williams Foundation. K. Phadwal was funded by the NIHR Biomedical Research Centre, Oxford. A. Williams, I. Fearnley, L. Rosser, J. Lowndes, C. Fratter, D. Ferguson, L. Vay, G. Quaghebeur, I. Moroni, S. Bianchi, C. Lamperti, S. Downes, K. Sitarz, and P. Flannery report no disclosures relevant to the manuscript. J. Carver was funded by the MRC (MR/J010448/1). E. Dombi was funded by the Lily Foundation, the Angus Memorial Mitochondrial Fund, and the NewLife Foundation. D. East, M. Laura, M. Reilly, H. Mortiboys, R. Prevo, and M. Campanella report no disclosures relevant to the manuscript. M. Daniels reports sponsorship from the Wellcome Trust (WT098519MA). M. Zeviani reports no disclosures relevant to the manuscript. P. Yu-Wai-Man holds a consultancy agreement with GenSigh Biologics (Paris, France). A. Katharina Simon was funded by the NIHR Biomedical Research Centre, Oxford. M. Votruba reports no disclosures relevant to the manuscript. J. Poulton was funded by the Wellcome Trust (0948685/Z/10/Z), the MRC (MR/J010448/1), Lily Foundation, the Angus Memorial Mitochondrial Fund, and the NewLife Foundation. Go to Neurology.org for full disclosures.

Received July 5, 2016. Accepted in final form October 4, 2016.

\section{REFERENCES}

1. Gomes LC, Di Benedetto G, Scorrano L. During autophagy, mitochondria elongate are spared from degradation and sustain cell viability. Nat Cell Biol 2011;13:589-598.

2. Klionsky DJ, Abdelmohsen K, Abe A, et al. Guidelines for the use and interpretation of assays for monitoring autophagy (3rd edition). Autophagy 2016;12:1-222.

3. Rugarli EI, Langer T. Mitochondrial quality control: a matter of life and death for neurons. EMBO J 2012; 31:1336-1349.
4. Mortensen M, Ferguson DJ, Edelmann M, et al. Loss of autophagy in erythroid cells leads to defective removal of mitochondria and severe anemia in vivo. Proc Natl Acad Sci USA 2010;107:832-837.

5. Dai Y, Zheng K, Clark J, et al. Rapamycin drives selection against a pathogenic heteroplasmic mitochondrial DNA mutation. Hum Mol Genet 2014;23:637-647.

6. Dombi E, Diot A, Morten K, et al. The m.13051G $>$ A mitochondrial DNA mutation results in variable neurology and activated mitophagy. Neurology 2016;86: 1921-1923.

7. Amati-Bonneau P, Guichet A, Olichon A, et al. OPA1 $\mathrm{R} 445 \mathrm{H}$ mutation in optic atrophy associated with sensorineural deafness. Ann Neurol 2005;58:958-963.

8. Carelli V, Musumeci O, Caporali L, et al. Syndromic parkinsonism and dementia associated with OPA1 missense mutations. Ann Neurol 2015;78:21-38.

9. Longatti A, Orsi A, Tooze SA. Autophagosome formation: not necessarily an inside job. Cell Res 2010;20: 1181-1184.

10. Korolchuk VI, Saiki S, Lichtenberg M, et al. Lysosomal positioning coordinates cellular nutrient responses. Nat Cell Biol 2011;13:453-460.

11. Pesch UE, Leo-Kottler B, Mayer S, et al. OPA1 mutations in patients with autosomal dominant optic atrophy and evidence for semi-dominant inheritance. Hum Mol Genet 2001;10:1359-1368.

12. Schaaf CP, Blazo M, Lewis RA, et al. Early-onset severe neuromuscular phenotype associated with compound heterozygosity for OPA1 mutations. Mol Genet Metab 2011; 103:383-387.

13. Yu-Wai-Man P, Griffiths PG, Gorman GS, et al. Multisystem neurological disease is common in patients with OPA1 mutations. Brain 2010;133:771-786.

14. Amati-Bonneau P, Valentino ML, Reynier P, et al. OPA1 mutations induce mitochondrial DNA instability and optic atrophy "plus" phenotypes. Brain 2008; 131:338-351.

15. Yu-Wai-Man P, Sitarz KS, Samuels DC, et al. OPA1 mutations cause cytochrome c oxidase deficiency due to loss of wild-type mtDNA molecules. Hum Mol Genet 2010; 19:3043-3052

16. Diot A, Hinks-Roberts A, Lodge T, et al. A novel quantitative assay of mitophagy: combining high content analysis fluorescence with mitochondrial DNA mutant load to identify novel pharmacological modulators of mitophagy. Pharmacol Res 2015;100:24-35.

17. Sarzi E, Angebault C, Seveno M, et al. The human OPAIdelTTAG mutation induces premature agerelated systemic neurodegeneration in mouse. Brain 2012;135:3599-3613.

18. Ashley N, Harris D, Poulton J. Detection of mitochondrial DNA depletion in living human cells using PicoGreen staining. Exp Cell Res 2005;303:432-446.

19. Uusimaa J, Evans J, Smith C, et al. Clinical, biochemical, cellular and molecular characterization of mitochondrial DNA depletion syndrome due to novel mutations in the MPV17 gene. Eur J Hum Genet 2014;22:184-191.

20. Elachouri G, Vidoni S, Zanna C, et al. OPA1 links human mitochondrial genome maintenance to mtDNA replication and distribution. Genome Res 2011;21:12-20.

21. Ashley N, Poulton J. Anticancer DNA intercalators cause p53-dependent mitochondrial DNA nucleoid re-modelling. Oncogene 2009;28:3880-3891. 
22. Kimura S, Noda T, Yoshimori T. Dynein-dependen movement of autophagosomes mediates efficient encounters with lysosomes. Cell Struct Funct 2008;33: 109-122.

23. Korolchuk VI, Rubinsztein DC. Regulation of autophagy by lysosomal positioning. Autophagy 2011;7:927-928.

24. Ebneth A, Godemann R, Stamer K, Illenberger S, Trinczek B, Mandelkow E. Overexpression of tau protein inhibits kinesin-dependent trafficking of vesicles, mitochondria, and endoplasmic reticulum: implications for Alzheimer's disease. J Cell Biol 1998;143:777-794.

25. Gegg ME, Cooper JM, Chau KY, Rojo M, Schapira AH, Taanman JW. Mitofusin 1 and mitofusin 2 are ubiquitinated in a PINK1/parkin-dependent manner upon induction of mitophagy. Hum Mol Genet 2010;19:4861-4870.

26. Narendra DP, Jin SM, Tanaka A, et al. PINK1 is selectively stabilized on impaired mitochondria to activate Parkin. PLoS Biol 2010;8:e1000298.

27. Narendra D, Tanaka A, Suen DF, Youle RJ. Parkin is recruited selectively to impaired mitochondria and promotes their autophagy. J Cell Biol 2008;183:795-803.

28. Carelli V, Sabatelli M, Carrozzo R, et al. "Behr syndrome" with OPA1 compound heterozygote mutations. Brain 2015;138:e321.

29. Mishra P, Carelli V, Manfredi G, Chan DC. Proteolytic cleavage of Opal stimulates mitochondrial inner membrane fusion and couples fusion to oxidative phosphorylation. Cell Metab 2014;19:630-641.

30. Olichon A, Landes T, Arnaune-Pelloquin L, et al. Effects of OPA1 mutations on mitochondrial morphology and apoptosis: relevance to ADOA pathogenesis. J Cell Physiol 2007;211:423-430.
31. Civiletto G, Varanita T, Cerutti R, et al. Opa1 overexpression ameliorates the phenotype of two mitochondrial disease mouse models. Cell Metab 2015;21:845-854.

32. Bertholet AM, Millet AM, Guillermin O, et al. OPA1 loss of function affects in vitro neuronal maturation. Brain 2013;136:1518-1533.

33. Gilkerson RW, De Vries RL, Lebot P, et al. Mitochondrial autophagy in cells with mtDNA mutations results from synergistic loss of transmembrane potential and mTORC1 inhibition. Hum Mol Genet 2012;21:978-990.

34. White KE, Davies V, Hogan V, et al. OPA1 deficiency is associated with increased autophagy in retinal ganglion cells in a murine model of dominant optic atrophy. Invest Ophthalmol Vis Sci 2009;50:2567-2571.

35. Kim TY, Wang D, Kim AK, et al. Metabolic labeling reveals proteome dynamics of mouse mitochondria. Mol Cell Proteomics 2012;11:1586-1594.

36. Twig G, Elorza A, Molina AJ, et al. Fission and selective fusion govern mitochondrial segregation and elimination by autophagy. EMBO J 2008;27:433-446.

37. Lemasters JJ. Variants of mitochondrial autophagy: types 1 and 2 mitophagy and micromitophagy (type 3). Redox Biol 2014;2:749-754.

38. Baloh RH, Schmidt RE, Pestronk A, Milbrandt J. Altered axonal mitochondrial transport in the pathogenesis of Charcot-Marie-Tooth disease from mitofusin 2 mutations. J Neurosci 2007;27:422-430.

39. Tondera D, Grandemange S, Jourdain A, et al. SLP-2 is required for stress-induced mitochondrial hyperfusion. EMBO J 2009;28:1589-1600.

40. Giordano C, Iommarini L, Giordano L, et al. Efficient mitochondrial biogenesis drives incomplete penetrance in Leber's hereditary optic neuropathy. Brain 2014;137:335-353.

\section{Visit the Neurology ${ }^{\circledR}$ Resident \& Fellow Website}

Click on Residents \& Fellows tab at Neurology.org.

Now offering:

- Neurology ${ }^{\circledR}$ Resident \& Fellow Editorial team information

- "Search by subcategory" option

- E-pearl of the Week

- RSS Feeds

- Direct links to Continuum ${ }^{\circledR}$, Career Planning, and AAN Resident \& Fellow pages

- Recently published Resident \& Fellow articles

- Podcast descriptions

(A :

twitter Follow Neurology ${ }^{\circledR}$ on Twitter: http://twitter.com/GreenJournal 


\section{Neurology}

\section{Dysregulated mitophagy and mitochondrial organization in optic atrophy due to $O P A I$ mutations}

Chunyan Liao, Neil Ashley, Alan Diot, et al.

Neurology 2017;88;131-142 Published Online before print December 14, 2016

DOI 10.1212/WNL.0000000000003491

\section{This information is current as of December 14, 2016}

\section{Updated Information \& Services}

Supplementary Material

\section{References}

Citations

Subspecialty Collections

Permissions \& Licensing

Reprints including high resolution figures, can be found at: http://n.neurology.org/content/88/2/131.full

Supplementary material can be found at: http://n.neurology.org/content/suppl/2016/12/14/WNL.0000000000003 491.DC1

This article cites 40 articles, 11 of which you can access for free at: http://n.neurology.org/content/88/2/131.full\#ref-list-1

This article has been cited by 1 HighWire-hosted articles: http://n.neurology.org/content/88/2/131.full\#\#otherarticles

This article, along with others on similar topics, appears in the following collection(s):

\section{Mitochondrial disorders}

http://n.neurology.org/cgi/collection/mitochondrial_disorders Mitochondrial disorders; see Genetics/Mitochondrial disorders http://n.neurology.org/cgi/collection/mitochondrial_disorders_see_gene tics-mitochondrial_disorders

Information about reproducing this article in parts (figures,tables) or in its entirety can be found online at:

http://www.neurology.org/about/about_the_journal\#permissions

Information about ordering reprints can be found online:

http://n.neurology.org/subscribers/advertise

Neurology ${ }^{\circledR}$ is the official journal of the American Academy of Neurology. Published continuously since 1951, it is now a weekly with 48 issues per year. Copyright Copyright ( 2016 The Author(s). Published by Wolters Kluwer Health, Inc. on behalf of the American Academy of Neurology. All rights reserved. Print ISSN: 0028-3878. Online ISSN: 1526-632X.

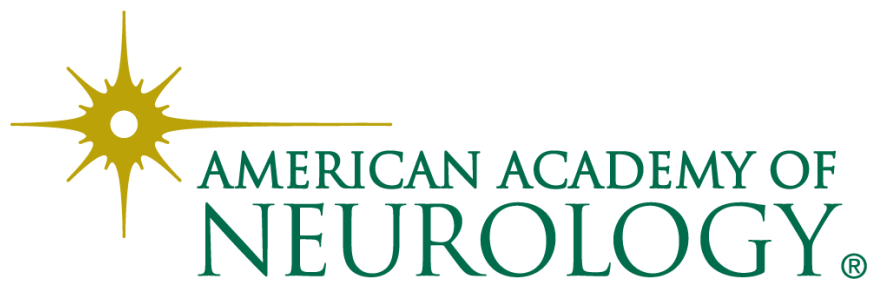

